\title{
Distinct expression profile of key molecules in crawling-type early gastric carcinoma
}

\author{
Ha Young Woo' ${ }^{1}$ Yoon Sung Bae ${ }^{1} \cdot$ Jie-Hyun $\mathrm{Kim}^{2} \cdot$ Sang Kil Lee ${ }^{3} \cdot$ \\ Yong Chan Lee ${ }^{3} \cdot$ Jae-Ho Cheong $^{4} \cdot$ Sung Hoon Noh $^{4} \cdot$ Hyunki Kim $^{1}[$
}

Received: 27 June 2016/ Accepted: 27 September 2016/Published online: 12 October 2016

(c) The International Gastric Cancer Association and The Japanese Gastric Cancer Association 2016

\begin{abstract}
Background Gastric "crawling-type" adenocarcinoma (CRA) is a tumor histologically characterized by irregularly fused glands with low-grade cellular atypia that tends to spread laterally in the mucosa. To date, the expression characteristics of the key molecules involved in CRA, including receptor tyrosine kinases (RTKs), mismatch repair (MMR) proteins, phosphatase and tensin homolog (PTEN), as well as the Epstein-Barr virus (EBV) status, have yet to be uncovered.

Methods We constructed tissue microarrays of 94 CRAs, 72 conventional-type differentiated adenocarcinomas (CDAs), and 71 intramucosal poorly cohesive adenocarcinomas (PCAs) from early gastric cancers to evaluate and compare the pathological and expression profiles of potential key molecules for molecular classification (EBV; four MMR proteins-MLH1, MSH2, PMS2, and MSH6; three RTKs-HER2, MET, and EGFR; PTEN; and p53).
\end{abstract}

Electronic supplementary material The online version of this article (doi:10.1007/s10120-016-0652-y) contains supplementary material, which is available to authorized users.

Hyunki Kim

kimhyunki@yuhs.ac

1 Department of Pathology, Yonsei University College of Medicine, 50 Yonsei-ro, Seodaemun-gu, Seoul 120-752, Republic of Korea

2 Division of Gastroenterology, Department of Internal Medicine, Gangnam Severance Hospital, Yonsei University College of Medicine, Seoul, Republic of Korea

3 Division of Gastroenterology, Department of Internal Medicine, Severance Hospital, Yonsei University College of Medicine, Seoul, Republic of Korea

4 Department of Surgery, Yonsei University College of Medicine, Seoul, Republic of Korea
Results None of the CRAs showed MMR deficiency $(0.0 \%$ vs. $5.6 \%$, CRA vs. CDA, $p=0.036)$, HER2 overexpression $(0.0 \%$ vs. $12.5 \%, p=0.001)$, or loss of PTEN expression ( $0.0 \%$ vs. $9.7 \%, p=0.003)$. Moreover, MET overexpression (4.4\% vs. $19.4 \%, p=0.004$ ), and a mutant p53 pattern $(12.4 \%$ vs. $62.5 \%, p<0.001)$ were significantly less common in CRAs than in CDAs. However, clinicopathological features and all the profile of the molecules of CRAs were close to those of the PCA group. Conclusions CRA demonstrated unique clinicopathological characteristics and showed a distinct expression profile of key molecules, which was close to that of a null phenotype. These results support the classification of CRA as a distinct subgroup of gastric adenocarcinoma.

Keywords Gastric cancer - Crawling-type adenocarcinoma $\cdot$ Protein expression profile

\section{Introduction}

Crawling-type adenocarcinoma (CRA) is a rare variant of gastric cancer (GC) that is characterized by low-grade nuclear atypia and irregularly fused glands $[1,2]$. The characteristic histological details are as follows: (1) cytologically low-grade or extremely well-differentiated tumor cells resembling intestinal metaplasia; (2) growth in architecturally tortuous, branching, anastomosing, distending, abortive and spiky patterns with glandular outgrowth; and (3) topographically, tumor glands distributed predominantly in the middle third of the stomach with occasional signet-ring cells [2]. In prior literature, the distinguishing architectural features were referred to as a "shaking-hands structure," "WHYX pattern," and "crawling type" $[1,3,4]$. Because the neoplastic cells of 
CRA show minimal cellular atypia with extension into epithelial proliferative zone but with sparing of the mucosal surface, making an accurate diagnosis has been a great challenge, especially on biopsy specimens. As a result, this type is often misdiagnosed as indeterminate for neoplasia or as reactive intestinal metaplasia [2]. The borders of intramucosal CRA are frequently poorly defined because of lack of contrast from the surrounding nonneoplastic mucosa. This feature of CRA often results in the failure of complete resection after an endoscopic submucosal dissection (ESD) [5].

Some authors described CRA as extremely well-differentiated intestinal type $[5,6]$ or very well differentiated gastric adenocarcinoma of intestinal type [2], which emphasizes the bland nuclear features. However, the bland cytological features of CRA and this nomenclature might be misinterpreted, leading to an underestimation of its malignancy potential. Okamoto et al. [1] noted the frequent presence of a poorly cohesive component within the submucosal layer in the case of a submucosal invasive CRA. The authors therefore suggested that CRA possesses a more aggressive biological behavior than previously thought. Thus, the true biological behavior of CRA still remains to be elucidated. However, the rarity of this variant, which comprises only $1.9 \%$ of early GC, makes it difficult to evaluate its behavioral, clinicopathological, and molecular characteristics [2].

Recently, the cancer genome atlas (TCGA) project reported four molecular subtypes of GC based on comprehensive genomic, epigenomic, and transcriptomic analyses [7]. Unsupervised clustering of the data divided GC into four groups: (1) GC with Epstein-Barr virus (EBV) positivity in which DNA methylation, mutation of $P I K 3 C A$, and amplification of $P D-L 1$ were frequently observed; (2) GC with microsatellite instability (MSI), characterized by silencing of mismatch-repair (MMR) genes, such as MLH1, and elevated mutation rates; (3) GC with chromosomal instability (CIN), which showed marked aneuploidy, amplifications of receptor tyrosine kinases (RTKs), including HER2, EGFR, FGFR, MET, ERBB3, and frequent TP53 mutations; and (4) GC with genomic stability (GS), demonstrating an increased prevalence of the diffuse type, mutations of $R H O A$, or translocation of the $R H O$ family of genes.

In this study, we first evaluated the clinicopathological features of CRA using a large series of CRA cohort. To clarify the molecular characteristics of CRA, we selected potential key molecules that might be expected to be enable to classify CRA into one of the four molecular subtypes reported by the TCGA. These key proteins and molecules were EBV, MMR proteins, several RTKs, including HER2, EGFR and MET, PTEN, and a CIN group-related protein, p53. We then obtained the results using immunohistochemistry (IHC) and in situ hybridization (ISH) methods, which are clinically feasible assay platforms.

\section{Materials and methods}

\section{Patients and tissue collection}

We selected 94 cases of CRAs, which were obtained from either surgical resections $(n=5)$ or endoscopic submucosal dissections $(n=89)$ performed from 2008 to 2015 . All cases met the histological criteria, as previously proposed $[2,4,8]$. The criteria that we used were a tumor composed of neoplastic epithelium with low-grade nuclear atypia with differentiation toward metaplastic intestinaltype cells. Architecturally, glandular structures were typically tortuous, branching, or anastomosing, and resemble the shapes of the letters $\mathrm{W}, \mathrm{H}, \mathrm{Y}$, or X on low-power view. The neoplastic glands were usually sparsely distributed and lacked back-to-back gathering. All 94 cases were reviewed by three pathologists (H.K.K., Y.S.B., H.Y.W.) to confirm the diagnosis of CRA. As control groups, we collected 72 consecutive cases of gastric conventional differentiated adenocarcinomas (CDAs) between 2008 and 2012 and 71 consecutive cases of intramucosal poorly cohesive adenocarcinoma (PCA) between 2009 and 2015 collected from endoscopic submucosal dissections. This study was approved by the Institutional Review Board of Yonsei University College of Medicine (approval number: 4-20160088).

\section{Tissue microarray construction}

Two representative 3-mm-diameter tumor tissue cores from each formalin-fixed, paraffin-embedded primary tumor were assembled into tissue microarray (TMA) blocks. In the submucosal invasive case, a full thickness of mucosal layer and most of the submucosal component were sampled. Each TMA block contained 14 tumors and 1 normal gastric mucosal tissue core as a landmark and internal control. Then, 4- $\mu$ m-thick sections from each TMA block were prepared for immunohistochemistry (IHC) analysis.

\section{Epstein-Barr virus-encoded RNA in situ hybridization (EBER ISH)}

Epstein-Barr virus-encoded RNA in situ hybridization (EBER ISH) was performed with a Ventana Bench Mark system (ISH iView kit; Ventana, Tucson, AZ, USA). Paraffin-embedded tissue sections were deparaffinized with EZ Prep buffer (Ventana) and digested with protease I for 
$4 \mathrm{~min}$. Then, probes for EBER were denatured at $85{ }^{\circ} \mathrm{C}$ for $10 \mathrm{~min}$ and then hybridized at $37^{\circ} \mathrm{C}$ for $1 \mathrm{~h}$. After hybridization, the tissues were washed with $2 \times$ SSC buffer at $57{ }^{\circ} \mathrm{C}$. Incubation with anti-fluorescein monoclonal antibodies was then performed for $20 \mathrm{~min}$, and then an Alkaline Blue detection kit (Ventana) was used according to the manufacturer's protocol. The slides were counterstained with Nuclear Fast Red for 10 min.

\section{Immunohistochemistry}

Immunohistochemistry (IHC) was performed using a Ventana XT automated stainer (Ventana) with antibodies for MutL homolog 1 (MLH1, ready to use, clone M1, Roche, Basel, Switzerland), MutS protein homolog 2 (MSH2, ready to use, clone G219-1129; Roche), MutS homolog 6 (MSH6, 1:100, clone 44; Cell Marque, Rocklin, CA, USA), postmeiotic segregation increased 2 (PMS2, 1:40, clone MRQ28; Cell Marque), epidermal growth factor receptor 2 (HER2, ready to use, clone 4B5; Roche), MET (ready to use, clone SP44; Roche), epidermal growth factor receptor (EGFR, 1:100, clone EP38Y; Abcam, Cambridge, UK), phosphatase and tensin homolog (PTEN, 1:100, clone 138G6; Cell Signaling, Danvers, MA, USA), and p53 (1:300, clone DO7; Novocastra, Newcastle, UK). IHC was performed in all cases as previously described [14].

\section{Evaluation of IHC}

HER2 staining was analyzed according to the system used in the TOGA trial and categorized using the following scale: 0 (no reactivity or membranous staining in $<5$ cells), $1+$ (faint/barely perceptible membrane staining in $\geq 5$ cells), $2+$ (weak to moderate complete or basolateral membrane staining in $\geq 5$ cells) and $3+$ (moderate to strong complete or basolateral membrane staining in $\geq 5$ cells) [9]. In this study, we regarded the TMA cores as biopsies rather than resection specimens. To assess the expression levels of MET and EGFR, we compared their intensities with that of non-neoplastic epithelial cells and scored them as: 0 (negative), 1+ (weaker or the same as non-neoplastic epithelial cells), $2+$ (moderately stronger than non-neoplastic epithelial cells, $3+$ (markedly stronger than nonneoplastic epithelial cells). For HER2, EGFR, and MET expression, cases scoring $2+$ or more were defined as overexpression cases. For MMR proteins (MLH1, MSH2, PMS2, and MSH6), no nuclear staining in all the neoplastic cells was designated as a loss of expression, whereas normal expression was defined as the presence of nuclear expression in tumor cells, irrespective of the proportion or intensity. In terms of PTEN evaluation, histology
(H) scores were applied as follows according to nuclear or cytoplasmic staining: 0 (negative), $1+$ (weak), $2+$ (moderate), and $3+$ (strong). The percentage of cells at different staining intensities was determined by visual assessment, with the score calculated using the formula $1 \times(\%$ of $1+$ cells $)+2 \times(\%$ of $2+$ cells $)+3 \times(\%$ of $3+$ cells $)$. Samples were then classified as either showing a loss of (H-score $<100)$ or intact $(\geq 100)$ PTEN expression [10]. In regard to $\mathrm{p} 53$ protein expression, the nuclear staining was taken into account, and it was scored as follows: (1) wildtype pattern, in which the tumor showed patchy $(\leq 50 \%$ of tumor cells) and weak positivity, and (2) mutant pattern, in which the tumor showed diffuse and strong nuclear positivity ( $>50 \%$ of tumor cells) or complete loss of expression [11]. All IHC results were accessed by two independent pathologists (H.K.K. and H.Y.W.) blinded to each other and with no prior knowledge of the clinicopathological parameters.

\section{Silver in situ hybridization (SISH)}

HER2 SISH was performed on the Ventana Discovery XT system (Ventana), according to the manufacturer's protocols. The HER2 DNA probe (Ventana) was denatured at $95{ }^{\circ} \mathrm{C}$ for $12 \mathrm{~min}$ and hybridized at $52{ }^{\circ} \mathrm{C}$ for $2 \mathrm{~h}$. The CEP17 probe (Ventana) was denatured at $95{ }^{\circ} \mathrm{C}$ for $12 \mathrm{~min}$ and hybridized at $44{ }^{\circ} \mathrm{C}$ for $2 \mathrm{~h}$. To visualize the HER 2 probes, the slides were incubated with secondary antibodies conjugated with horseradish peroxidase (HRP), followed by HRP-catalyzed silver precipitation (black dots). For visualization of the CEP17 probes, a rabbit anti-DNP primary antibody was detected by a secondary antibody conjugated with alkaline phosphatase, and a naphthol/fast red stain was subsequently applied. The sections were then counterstained with hematoxylin II (modified Mayer hematoxylin) and a bluing reagent (Ventana).

Silver in situ hybridization (SISH) scoring was performed by counting the signals of 60 non-overlapping nuclei with clearly distinguishable borders, after which the mean gene copy number and HER2/CEP17 ratio were calculated by dividing the sum of the gene copy number by 60. HER2 amplification was defined as a HER2/CEP17 ratio greater than $2.0[9,12]$.

\section{Statistical analysis}

The clinical and pathological data were analyzed with IBM SPSS version 20.0 (IBM, Armonk, NY, USA). Pearson's chi-square test, Fisher's exact test, and independent twosample $t$ test were applied in the statistical analyses. Statistical significance was defined as $p<0.05$. 


\section{Results}

\section{Clinicopathological features of CRA}

The clinicopathological features of the CRA cases in comparison with the CDA and PCA groups are summarized in Table 1. Compared to CDAs, CRAs occurred more frequently in younger patients $(57.3 \pm 9.7$ years vs. $66.2 \pm 7.9$ years, mean $\pm \mathrm{SD}, p<0.001)$. The tumor sizes ranged from 0.1 to $8.4 \mathrm{~cm}$. CRAs were larger in size than CDAs $(1.9 \pm 1.3 \mathrm{~cm}$ vs. $1.5 \pm 0.9 \mathrm{~cm}$, mean $\pm \mathrm{SD}$, $p=0.019)$. The lateral resection margins were involved in 19 (11.4 \%, 15 CDAs and 4 CRAs) cases, and CRAs were more frequently associated with incomplete resection (16.9\% vs. $5.6 \%, p=0.047$ ) than CDAs in ESD cases. There was no significant difference in tumor location or invasion depth between the two groups. PCAs occurred more often in women in comparison to CDAs $(54.9 \%$ vs. $38.3 \%, p=0.041)$. Otherwise, the other clinicopathological features of CRAs were close to PCAs.

\section{Expression profile of CRA}

Representative IHC and EBER-ISH results of the key molecules of CRA and CDA groups are shown in Fig. 1. The comparisons of the expression profiles of the key molecules of the three groups are summarized in Table 2 . Of the total of 237 cases, none of the cases was positive on EBER-ISH analysis. Additionally, none of the CRAs and PCAs showed a loss of expression for any of the MMR proteins, whereas 4 of $72(5.6 \%)$ CDA cases revealed the loss of MMR protein expression $(p=0.036) ; 3$ showed loss of both MLH1 and PMS2, and in 1 case MSH2 and MSH6 were absent.

All CRAs and PCAs showed either negativity (79 cases, $89.8 \%$, and 62 cases, $87.3 \%$, respectively) or weak positivity ( 9 cases, $10.2 \%$, and 9 cases, $12.7 \%$, respectively) for HER2. On the other hand, 9 CDA cases $(12.5 \%)$ demonstrated HER2 overexpression ( $2+$ in 5 cases, $6.9 \%$; $3+$ in four cases, $5.6 \%)(p=0.001)$. Of these 9 cases, 8 $(88.9 \%)$ showed HER2 gene amplification on SISH analysis (Fig. 2). HER2 was not amplified in any of the cases with a negative or $1+$ score on IHC. MET overexpression was observed in only $4(4.4 \%$, all $2+)$ of CRA and 2 $(2.8 \%$, all $2+)$ of PCA group, whereas it was found in 14 $(19.4 \%, 3+$ in 1 and $2+$ in 13$)$ of CDA group (CRA vs. CDA, $p=0.004)$. None of the CRA and PCA cases showed a loss of PTEN expression. However, 7 (9.7 \%) CDAs had a calculated H-score below 100 (the actual scores being $80,70,65,60,30,0$, and 0 ), which was interpreted as loss of expression (CRA vs. CDA, $p=0.003$ ). EGFR overexpression was detected in 31 (36.5\%) of CRAs, 22 (31.4\%) of CDAs, and $22(31.0 \%)$ of PCAs (CRA vs. CDA, $p=0.610$ and CRA vs. PCA, $p=0.501)$. In all groups, the majority were stained with weak $(1+)$ or moderate $(2+)$ positivity $(1+$ in $48.2 \%$ of CRAs, $44.3 \%$ of CDAs, and $57.7 \%$ of PCAs; $2+$ in $35.3 \%$ of CRAs, $31.4 \%$ of CDAs, and $31.0 \%$ of PCAs).

Either diffuse and strong positive or complete loss of p53 expression was observed in $12.4 \%$ (11/89) of CRA group (diffuse and strong in 3 and complete loss in 8 cases) and $8.5 \%(6 / 71)$ of PCAs (diffuse and strong in 3 and

Table 1 Clinicopathological features of crawling-type (CRA), conventional differentiated (CDA), and poorly cohesive adenocarcinoma (PCA)

\begin{tabular}{|c|c|c|c|c|c|}
\hline & CRA $(\%)(N=94)$ & $\operatorname{CDA}(\%)(N=72)$ & $\operatorname{PCA}(\%)(N=71)$ & CRA vs. CDA & CRA vs. PCA \\
\hline Age (years, mean \pm SD) & $57.3 \pm 9.7$ & $66.2 \pm 7.9$ & $54.8 \pm 12.4$ & $<0.001^{*}$ & 0.167 \\
\hline Gender & & & & 0.094 & 0.041 \\
\hline Male & $58(61.7)$ & $54(75.0)$ & $32(45.1)$ & & \\
\hline Female & $36(38.3)$ & $18(25.0)$ & $39(54.9)$ & & \\
\hline Size $(\mathrm{cm}$, mean $\pm \mathrm{SD})$ & $1.9 \pm 1.3$ & $1.5 \pm 0.9$ & $1.7 \pm 0.9$ & $0.019 *$ & 0.428 \\
\hline Location & & & & 0.15 & 0.344 \\
\hline Lower third & $56(59.6)$ & $53(73.6)$ & $35(49.3)$ & & \\
\hline Middle third & $36(38.3)$ & $18(25.0)$ & $33(46.5)$ & & \\
\hline Upper third & $2(2.1)$ & $1(1.4)$ & $3(4.2)$ & & \\
\hline Invasion depth & & & & 0.635 & N/A \\
\hline Lamina propria & $59(62.8)$ & $40(55.6)$ & $59(83.1)$ & & \\
\hline Muscularis mucosa & $27(28.7)$ & $26(36.1)$ & $12(16.9)$ & & \\
\hline Submucosa & $8(8.5)$ & $6(8.3)$ & - & & \\
\hline Resection margin & & & & 0.047 & 0.37 \\
\hline Not involved & $74(83.1)$ & $68(94.4)$ & $63(88.7)$ & & \\
\hline Involved & $15(16.9)$ & $4(5.6)$ & $8(11.3)$ & & \\
\hline
\end{tabular}



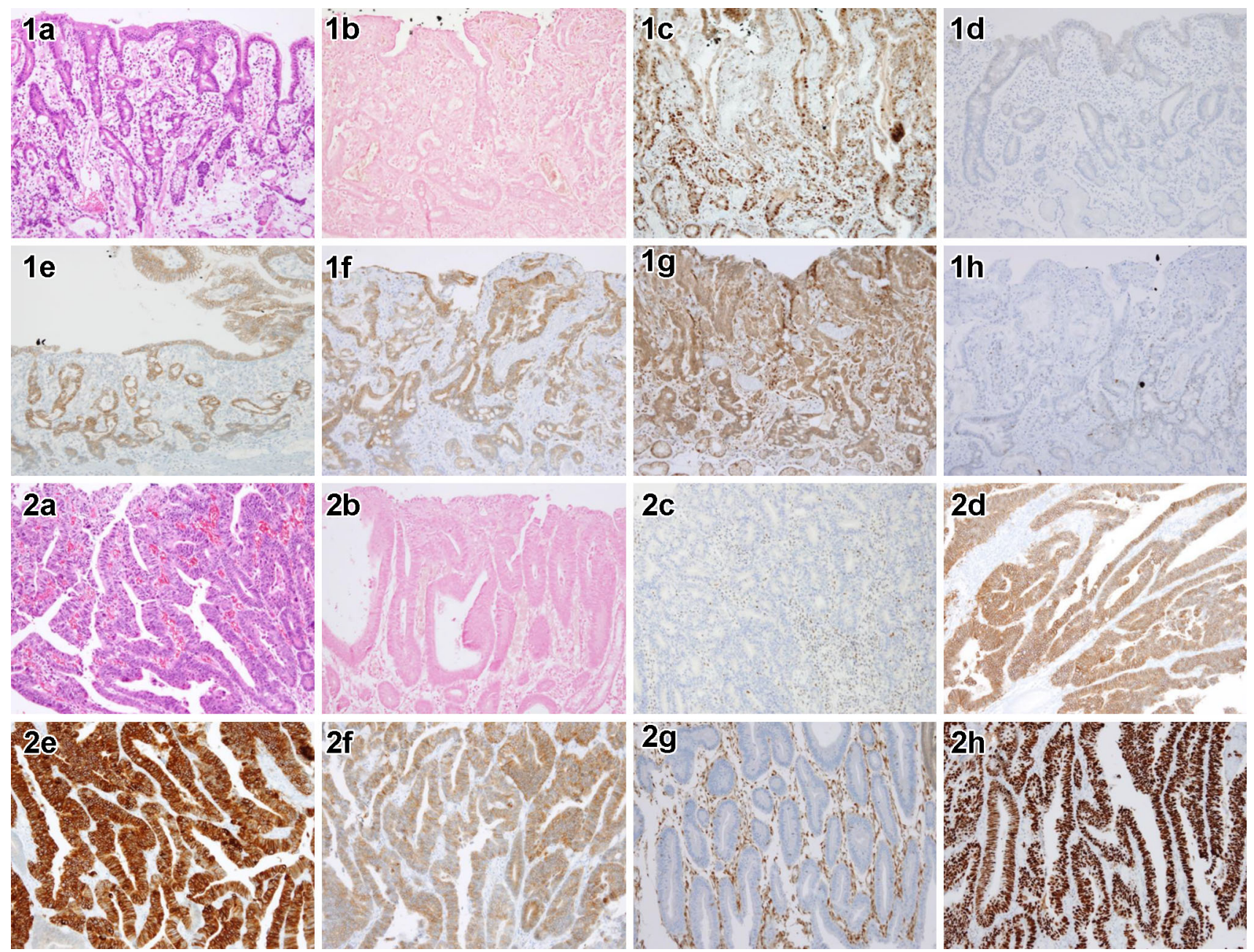

Fig. 1 Epstein-Barr virus-encoded RNA in situ hybridization (EBER ISH) and immunochemistry (IHC) results in crawling-type adenocarcinoma (CRA) and conventional differentiated adenocarcinoma (CDA). (1) Representative images of CRA [hematoxylin and eosin (H\&E)] (1-a). CRA shows negativity for EBER-ISH (1-b), positivity for MMR protein, e.g., MLH1 (1-c), negativity for HER2 (1-d), 1+ for MET (1-e), 2+ for EGFR (1-f), intact PTEN expression (1-g), and

complete loss in 3 cases), in contrast to $62.5 \%$ (45/72) of CDAs (diffuse and strong in 23 and complete loss in 22 cases) (CRA vs. CDA, $p<0.001$ ).

Twenty-four cases of CRAs $(25.5 \%, 24 / 94)$ showed a minor component of poorly differentiated (PD) adenocarcinoma component in the tumor. There were no significantly different expression profiles between the CRAs with and without PD component, as shown in Supplementary Table 1.

\section{Discussion}

CRA was first described as a neoplasm "mimicking intestinal metaplasia," by Endoh et al. [4], and later Yao et al. reported 9 additional cases of "extremely well-
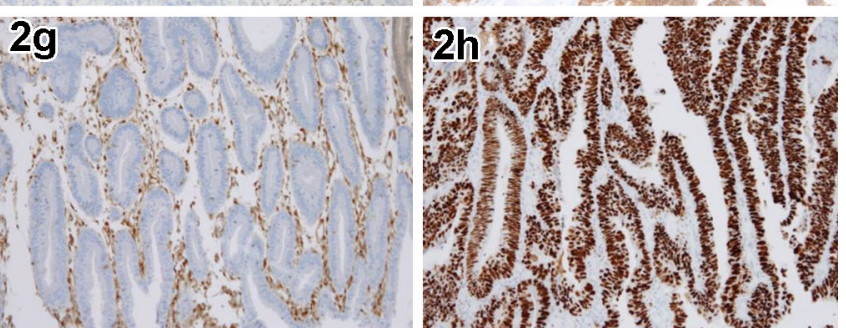

wild-type p53 pattern (1-h). (2) Representative CDA images (H\&E) (2-a). CDAs are negative for EBER (2-b). Some of them show loss of MMR protein expression, e.g., MLH1 (2-c), 2+ for HER2 (2-d), 3+ for MET (2-e), 2+ for EGFR (2-f), loss of expression for PTEN (2-g), and diffuse strong positivity for p53 (2-h). H\&E, hematoxylin and eosin. $\times 100)$

differentiated adenocarcinoma" [6]. Okamoto et al. reported 25 cases of CRA with emphasis on the presence of poorly differentiated component in the submucosa where the tumor invaded [1]. Kushima et al. hypothesized that CRAs should be regarded as a "prediffuse type," despite their bland cytology and the presence of tubular components in the intramucosal layer [3]. Subsequently, Ushiku et al. presented a more detailed description of the histological, clinical, and immunohistochemical characteristics of 21 cases of "very well-differentiated gastric adenocarcinoma of intestinal type" [2]. They performed immunohistochemical stains for intestinal and gastric mucin markers and suggested that those with mixed immunophenotype behaved more aggressively, with a higher likelihood of dedifferentiating into a poorly cohesive carcinoma. During an endoscopic examination, CRA 
Table 2 EBER in situ hybridization (ISH) and immunohistochemistry results of crawling-type (CRA), conventional differentiated (CDA), and poorly cohesive adenocarcinoma (PCA)

\begin{tabular}{|c|c|c|c|c|c|}
\hline & CRA $(\%)(N=94)$ & $\operatorname{CDA}(\%)(N=72)$ & PCA $(\%)(N=71)$ & CRA vs. CDA & CRA vs. PCA \\
\hline \multicolumn{6}{|l|}{ EBER ISH } \\
\hline Positive & $0(0.0)$ & $0(0.0)$ & $0(0.0)$ & \multirow[t]{2}{*}{ N/A } & \multirow[t]{2}{*}{ N/A } \\
\hline Negative & $90(100.0)$ & $72(100.0)$ & $71(100.0)$ & & \\
\hline \multicolumn{6}{|l|}{ MMR proteins } \\
\hline Deficient & $0(0.0)$ & $4(5.6)$ & $0(0.0)$ & \multirow[t]{2}{*}{0.036} & \multirow[t]{2}{*}{ N/A } \\
\hline Proficient & $91(100.0)$ & $68(94.4)$ & $71(100.0)$ & & \\
\hline \multicolumn{6}{|l|}{ HER2 } \\
\hline 2 or $3+$ & $0(0.0)$ & $9(12.5)$ & $0(0.0)$ & \multirow[t]{2}{*}{0.001} & \multirow[t]{2}{*}{ N/A } \\
\hline 0 or $1+$ & $88(100.0)$ & 63 (87.5) & $71(100.0)$ & & \\
\hline \multicolumn{6}{|l|}{ MET } \\
\hline 2 or $3+$ & $4(4.4)$ & 14 (19.4) & $2(2.8)$ & \multirow[t]{2}{*}{0.004} & \multirow[t]{2}{*}{0.695} \\
\hline 0 or $1+$ & 86 (95.6) & 58 (80.6) & 69 (97.2) & & \\
\hline \multicolumn{6}{|l|}{ EGFR } \\
\hline 2 or $3+$ & $31(36.5)$ & $22(31.4)$ & $22(31.0)$ & \multirow[t]{2}{*}{0.61} & \multirow[t]{2}{*}{0.501} \\
\hline 0 or $1+$ & $54(63.5)$ & $48(68.6)$ & $49(69.0)$ & & \\
\hline \multicolumn{6}{|l|}{ PTEN } \\
\hline Loss & $0(0.0)$ & $7(9.7)$ & $0(0.0)$ & \multirow[t]{2}{*}{0.003} & \multirow[t]{2}{*}{ N/A } \\
\hline Intact & $90(100.0)$ & $65(90.3)$ & $71(100.0)$ & & \\
\hline \multicolumn{6}{|l|}{ p53 } \\
\hline Mutant pattern & $11(12.4)$ & $45(62.5)$ & $6(8.5)$ & \multirow[t]{2}{*}{$<0.001$} & \multirow[t]{2}{*}{0.454} \\
\hline Wild pattern & 78 (87.6) & $27(37.5)$ & 65 (91.5) & & \\
\hline
\end{tabular}
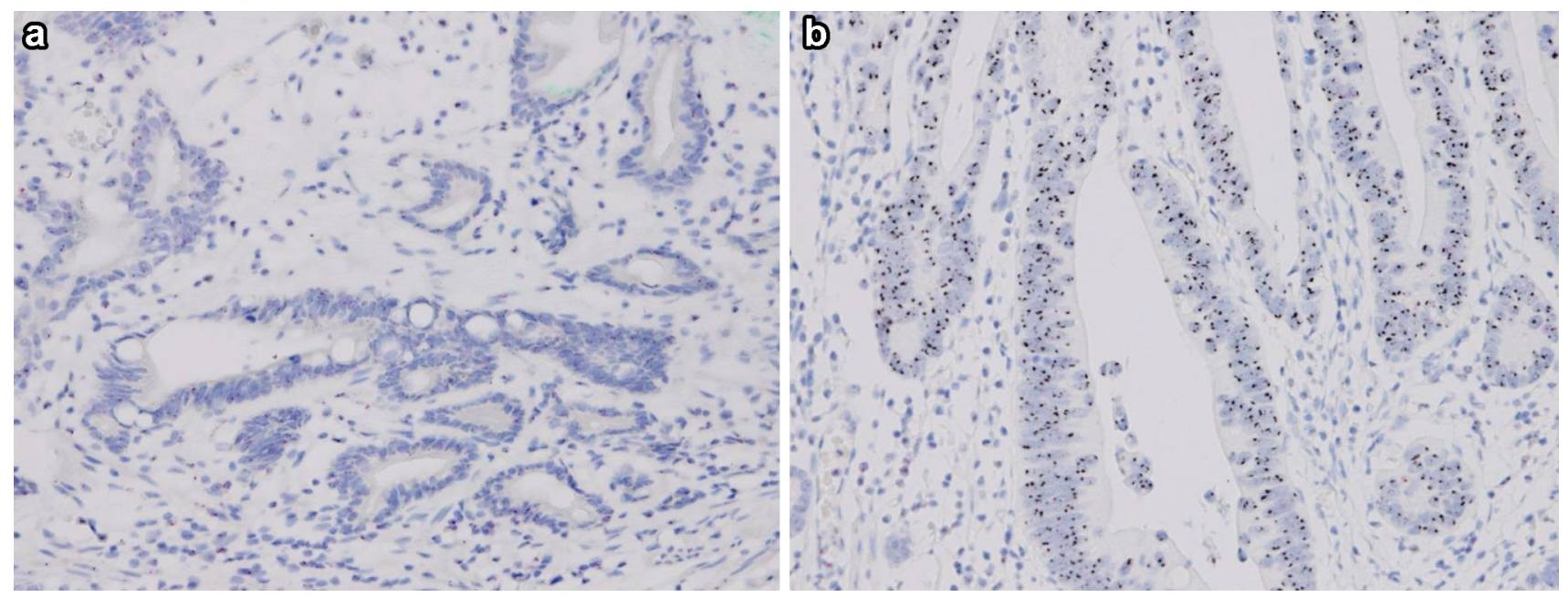

Fig. 2 HER2 SISH results in crawling-type adenocarcinoma (CRA) and conventional differentiated adenocarcinoma (CDA). Representative case of CRA shows non-amplification for HER2 $(\mathbf{a})$ and CDA case with HER2 amplification (allelic imbalance $=4.05)(\mathbf{b}) . \times 200$

appears as an ill-defined mass with indistinct borders, which may be the result of the "crawl" of tumor glands into the epithelial proliferative zone under a preserved foveolar epithelium [1]. This problematic feature results in the higher rates of incomplete resection, especially along the lateral margins [5].
In this study, we collected a large series of CRA ( $n=94)$, CDA $(n=72)$, and PCA $(n=71)$ cases with the aim of clarifying the unique clinicopathological and molecular features of CRA based on IHC and SISH data. On clinicopathological comparison, we found a relationship between the CRA group and younger patient age, 


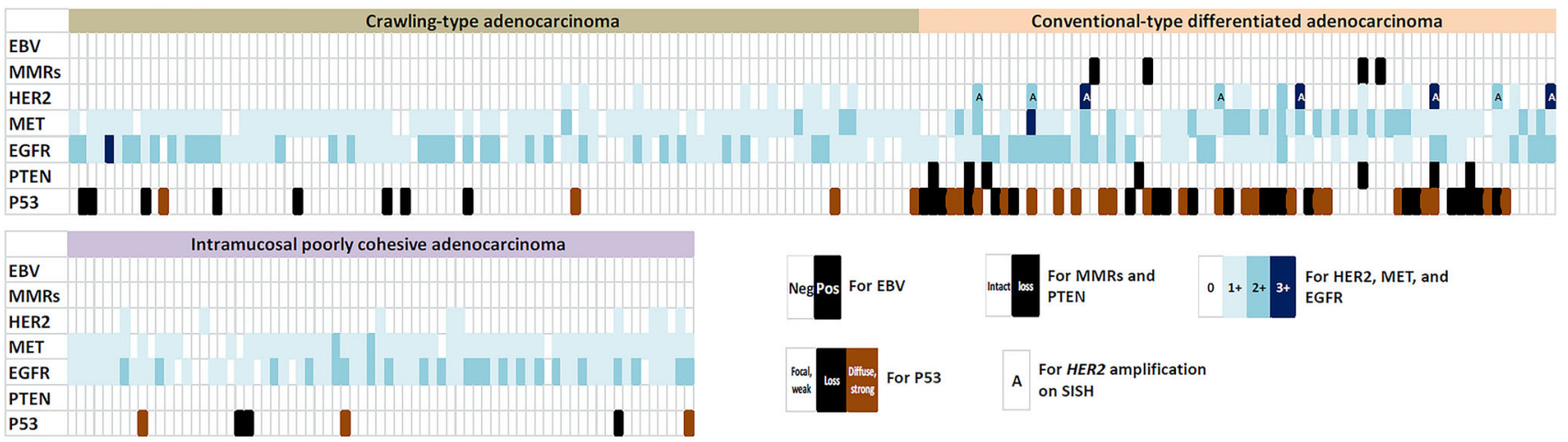

Fig. 3 Integrated expression profile of the key molecules. The expression profile of the key molecules in crawling-type adenocarcinoma (CRA), which shows a null phenotype for the molecules, is

confirming the findings of a previous report [5]. CRA tumors were also larger in size at the time of resection compared to CDA tumors, which again may be explained by "crawling" behavior of this type combined with the difficulty of making the diagnosis. As we expected, CRA was associated with the higher rates of incomplete resection (16\% vs. $5.6 \%$ for CRA and CDA, respectively), which is concordant with the previous reports [5].

The expression profiles of CRAs for EBV, MMR proteins, RTKs, PTEN, and p53 were close to a null phenotype, in comparison to CDA cases. This null phenotype of CRAs was similar with that of PCAs (Fig. 3). All cases in those three groups were negative in EBER-ISH analyses. It is possible that the poorly differentiated histology frequently seen in EBV-positive GC would have excluded these cases from enrollment in our study. None of the CRA cases was classified as MMR deficient. In this study, we did not perform genetic analysis for microsatellite instability (MSI); therefore, there may be discrepancies between the MMR protein results determined by IHC analysis and the MSI status. Nonetheless, several studies have reported a high concordance rate between the data from IHC evaluation for MMR proteins and the MSI status. In colon cancer, the concordance rate was as high as $98.6 \%$ [13]. We also recently validated the high sensitivity and specificity of the IHC approach for MSI detection in GC [14]. Thus, we believe that the IHC-obtained MMR protein results faithfully reflected the MSI status. We also evaluated the TP53 status via the IHC method in this study. In ovarian cancer, the p53 IHC result in cases showing complete loss or strong and diffuse expression in more than $60 \%$ of tumor cells was highly correlated with a TP53 mutation status $[11,15,16]$. Because the half-life of wildtype p53 protein is short (about $20 \mathrm{~min}$ ), the protein normally does not accumulate to be detectable by IHC analysis. However, the half-life of the mutant p53 protein is extended, thus allowing it to accumulate and be clearly different from those of conventional differentiated adenocarcinoma (CDA) and is more similar to those of poorly cohesive adenocarcinoma (PCA)

detectable by IHC method $[15,16]$. None of the CRA cases showed HER2 overexpression or loss of PTEN. In addition, other immunophenotypic changes, such as MET overexpression and demonstration of a mutant p53 pattern, were significantly less commonly observed in CRA, compared to CDA. The expression patterns of HER2 and p53 of CRAs were consistent with the findings by Yao et al. [6].

Among the four molecular groups described in the TCGA data, the GS type had the strongest association with diffuse-type histology, a younger age of onset, $\mathrm{CDH} 1$ mutations, and the lack of other key molecular alterations, including amplifications of HER2, EGFR, FGER2, MET, and KRAS and mutations of PIK3CA and TP53 [7]. Additionally, a considerable proportion of GS cancers harbor alterations in $R H O A$ and ROCKI gene expression. The modulation of RHOA and its downstream effectors, ROCKI and $M D I A$, may contribute to the lack of cell cohesion seen in the diffuse histological pattern [7, 17, 18]. GCs carrying the RHOA mutation have been reported to show a characteristically mixed histology with mucosal tubular tumor glands and poorly cohesive components in deeper areas [18]. The mixed histological feature of CRA that was described in studies published by Kushima et al. [3] and Okamoto et al. [1] was the basis for the hypothesis suggesting that CRA progresses into a diffuse-type GC, even though this type has a well-differentiated component in the mucosal area [1, 3]. Moreover, the null immunophenotype of CRAs and its resemblance to the molecular profiles of the PCA group implies CRAs may fall into the GS subtype of TCGA. Therefore, we hypothesized that CRA might be classified as a GS subtype of TCGA, given the younger age of onset, MMR proficiency, and lack of RTKs amplification and EBV negativity. A more comprehensive genomic analysis is needed to supplement our data.

In conclusion, CRA has a unique clinicomorphological and molecular phenotypic profile. According to our data, 
all or the majority of CRA cases showed no association with MMR protein deficiency, overexpression of RTKs, loss of PTEN, or mutant p53 pattern. We believe that our findings provide further supporting evidence for the findings published by Kushima et al. [3] and Okamoto et al. [1], that CRA be considered as a distinct entity and possibly an early stage of diffuse-type GC.

Acknowledgments This research was supported by the Basic Science Research Program through the National Research Foundation of Korea (NRF) and was funded by the Ministry of Science, ICT and Future Planning (2012R1A1A1004403) and by a faculty research Grant of Yonsei University College of Medicine for 2012 (6-20120044).

\section{Compliance with ethical standards}

Ethical standards All procedures followed were in accordance with the ethical standards of the responsible committee on human experimentation (the Institutional Review Board of Yonsei University College of Medicine, approval number: 4-2014-0667) and with the Helsinki Declaration of 1964 and later versions. Informed consent or substitute for it was obtained from all patients included in the study.

Conflict of interest The authors declare that they have no conflicts of interest.

\section{References}

1. Okamoto N, Kawachi H, Yoshida T, Kitagaki K, Sekine M, Kojima K, et al. "Crawling-type" adenocarcinoma of the stomach: a distinct entity preceding poorly differentiated adenocarcinoma. Gastric Cancer. 2013;16:220-32.

2. Ushiku T, Arnason T, Ban S, Hishima T, Shimizu M, Fukayama $\mathrm{M}$, et al. Very well-differentiated gastric carcinoma of intestinal type: analysis of diagnostic criteria. Mod Pathol. 2013;26:1620-31.

3. Kushima R, Vieth M, Borchard F, Stolte M, Mukaisho K, Hattori T. Gastric-type well-differentiated adenocarcinoma and pyloric gland adenoma of the stomach. Gastric Cancer. 2006;9:177-84.

4. Endoh Y, Tamura G, Motoyama T, Ajioka Y, Watanabe H. Welldifferentiated adenocarcinoma mimicking complete-type intestinal metaplasia in the stomach. Hum Pathol. 1999;30:826-32.

5. Kang KJ, Kim KM, Kim JJ, Rhee PL, Lee JH, Min BH, et al. Gastric extremely well-differentiated intestinal-type adenocarcinoma: a challenging lesion to achieve complete endoscopic resection. Endoscopy. 2012;44:949-52.

6. Yao T, Utsunomiya $\mathrm{T}$, Oya $\mathrm{M}$, Nishiyama $\mathrm{K}$, Tsuneyoshi $\mathrm{M}$. Extremely well-differentiated adenocarcinoma of the stomach: clinicopathological and immunohistochemical features. World $\mathrm{J}$ Gastroenterol. 2006;12:2510-6.

7. Comprehensive molecular characterization of gastric adenocarcinoma. Nature (Lond). 2014;513:202-9.

8. Kato Y, Yanagisawa A, Sugano H. Biopsy interpretation in diagnosis of gastric carcinoma. In: Nishi M, Ichikawa H, Nakajima T, et al, editors. Gastric Cancer. Tokyo: Springer; 1993. pp. 133-50.

9. Ruschoff J, Hanna W, Bilous M, Hofmann M, Osamura RY, Penault-Llorca F, et al. HER2 testing in gastric cancer: a practical approach. Mod Pathol. 2012;25:637-50.

10. Lotan TL, Gurel B, Sutcliffe S, Esopi D, Liu W, Xu J, et al. PTEN protein loss by immunostaining: analytic validation and prognostic indicator for a high risk surgical cohort of prostate cancer patients. Clin Cancer Res. 2011;17:6563-73.

11. Yemelyanova A, Vang R, Kshirsagar M, Lu D, Marks MA, Shih Ie $\mathrm{M}$, et al. Immunohistochemical staining patterns of p53 can serve as a surrogate marker for TP53 mutations in ovarian carcinoma: an immunohistochemical and nucleotide sequencing analysis. Mod Pathol. 2011;24:1248-53.

12. Papouchado BG, Myles J, Lloyd RV, Stoler M, Oliveira AM, Downs-Kelly E, et al. Silver in situ hybridization (SISH) for determination of HER2 gene status in breast carcinoma: comparison with FISH and assessment of interobserver reproducibility. Am J Surg Pathol. 2010;34:767-76.

13. Yuan L, Chi Y, Chen W, Chen X, Wei P, Sheng W, et al. Immunohistochemistry and microsatellite instability analysis in molecular subtyping of colorectal carcinoma based on mismatch repair competency. Int J Clin Exp Med. 2015;8:20988-1000.

14. Bae YS, Kim H, Noh SH, Kim H. Usefulness of immunohistochemistry for microsatellite instability screening in gastric cancer. Gut Liver. 2015;9:629-35.

15. Bennett WP, Hollstein MC, Hsu IC, Sidransky D, Lane DP, Vogelstein B, et al. Mutational spectra and immunohistochemical analyses of p53 in human cancers. Chest. 1992;101:19s-20s.

16. Lepelley P, Preudhomme C, Vanrumbeke M, Quesnel B, Cosson A, Fenaux P. Detection of p53 mutations in hematological malignancies: comparison between immunocytochemistry and DNA analysis. Leukemia. 1994;8:1342-9.

17. Thumkeo D, Watanabe S, Narumiya S. Physiological roles of Rho and Rho effectors in mammals. Eur $\mathrm{J}$ Cell Biol. 2013;92:303-15.

18. Kakiuchi M, Nishizawa T, Ueda H, Gotoh K, Tanaka A, Hayashi A, et al. Recurrent gain-of-function mutations of RHOA in diffuse-type gastric carcinoma. Nat Genet. 2014;46:583-7. 